ALVES JCS; PEIXOTO JR; VIEIRA JV; BOITEUX LS. 2006. Herdabilidade e correlações genotípicas entre caracteres de folhagem e sistema radicular em famílias de cenoura, cultivar Brasília. Horticultura Brasileira 24: 363-367.

\title{
Herdabilidade e correlações genotípicas entre caracteres de folhagem e sistema radicular em famílias de cenoura, cultivar Brasília
}

\author{
José Carlos da S Alves ${ }^{1}$; José Ricardo Peixoto ${ }^{1}$; Jairo V Vieira ${ }^{2}$; Leonardo S Boiteux ${ }^{2}$ \\ ${ }^{1}$ UnB, 70910-900 Brasília-DF; 2Embrapa Hortaliças, C. Postal 218, 70359-970 Brasília-DF; E-mail: jairo@cnph.embrapa.br
}

\begin{abstract}
RESUMO
O presente trabalho foi implementado com o objetivo de estimar parâmetros genéticos para os seguintes caracteres de importância para o melhoramento genético da cenoura: número de folhas por planta (NFP), comprimento da maior folha na planta (CF), comprimento da raiz (CR), peso da raiz (PR), diâmetro da raiz (DR) e diâmetro do xilema (DX). Este experimento foi conduzido em condições de campo durante o verão de 1999/2000, sendo utilizadas 69 famílias de meios-irmãos derivadas da cultivar Brasília. As progênies foram cultivadas em delineamento de blocos ao acaso com duas repetições e parcelas de $2 \mathrm{~m}^{2}$, com quatro linhas de 20 plantas cada. Os valores observados para coeficiente de variação genética oscilaram de 4,8 a 10,9 . Os valores calculados de herdabilidade no sentido amplo $\left(\mathrm{h}^{2}\right)$ foram medianamente altos para todos os caracteres, com uma variação observada entre $29,9 \%$ (para DR) e $77,6 \%$ (para CF). Foram também obtidas estimativas de valores de correlações fenotípicas, genotípicas e ambientais entre os caracteres avaliados, com destaque para a magnitude do valor da correlação genotípica entre PR e DR, de 0,85. Correlação genotípica negativa foi observada entre CR e DX indicando a possibilidade de desenvolver, a partir de populações derivadas de 'Brasília', novas cultivares com arquitetura de raiz mais adequada para a produção de mini-cenouras ("baby-carrots"). Valores de correlações ambientais negativos e altos foram observados entre PR e DX e entre DR e DX. Este conjunto de informações representa uma importante base de dados genéticos com implicações diretas na otimização do processo de seleção dentro de populações de cenoura derivadas da cultivar Brasília.
\end{abstract}

Palavras-chave: Daucus carota, herdabilidade, parâmetros genético, correlações, cultivo de verão.

\begin{abstract}
Heritability and genotypic correlation among leaf and root traits in carrot, cultivar Brasilia progenies

The present work was conducted aiming to estimate genetic parameters associated with traits of importance to carrot breeding, including: number of leaves per plant (NL), length of the longest leaf in the plant (LL), root length (RL), root weight (RW), root diameter (RD), and xylem diameter (XD). This experiment was carried out during the rainy summer season (1999/2000) using 69 half-sib families derived from cultivar Brasília. Progenies were evaluated using a complete randomized block design with two replications and $2 \mathrm{~m}^{2}$ plots, with four 20-plant rows. The broadsense heritability $\left(\mathrm{h}^{2}\right.$ ) values ranged from $29.9 \%$ (for RD) to $77.6 \%$ (for LL). Genotypic, phenotypic, and environmental correlations displayed a large variation in magnitude with the highest genotypic correlation (0.85) being observed between the traits RW and RD. Negative genotypic correlation was observed between RL and XD, which indicates that the development of new cultivars suitable for processing as baby-carrots is feasible using populations derived from cultivar Brasilia. High negative environmental correlation values were obtained between the traits RW and XD, as well as between $\mathrm{RD}$ and XD. This information would be of extreme importance aiming to optimize the selection process when using segregating populations derived from the tropical-adapted cultivar Brasilia.
\end{abstract}

Keywords: Daucus carota, genetic parameters, heritability, genetic correlation, summer cropping

(Recebido para publicação em 23 de fevereiro de 2006; aceito em 29 de agosto de 2006)

\begin{abstract}
$\mathrm{A}$ s cultivares modernas de cenoura (Daucus carota L.) foram desenvolvidas a partir de populações complexas, fenotipicamente heterogêneas e de base genética ampla, tendo sido submetidas a diversos ciclos de seleção, em diferentes regiões da Europa, antes de alcançar o atual estádio de domesticação (Vieira, 1988). Estas seleções foram muito provavelmente focadas em caracteres de planta e de raiz, sendo feitas via seleção massal (antes do florescimento) à semelhança dos processos de seleção conduzidos com cebola (Buso, 1978). O método de seleção massal tem se mostrado como uma das estratégias mais eficientes para o me-
\end{abstract}

lhoramento genético da cenoura, permitindo o desenvolvimento de inúmeras cultivares com adaptação a diferentes condições agroedafoclimáticas e combinando diversos atributos de interesse para o cultivo, comercialização, processamento e consumo desta hortaliça (Vieira et al., 1983; Casali et al., 1984; Rubatzky et al., 1999).

Dentro do contexto relativo ao processo de seleção, o conhecimento dos componentes de variância é de fundamental importância uma vez que propicia as condições para estimar a herdabilidade, predizer o ganho genético e avaliar as potencialidades de uma população e a eficiência relativa dos di- ferentes métodos de melhoramento, auxiliando, desta forma, a identificar a estratégia de seleção mais adequada (Hallauer \& Miranda Filho, 1981). Outro conjunto de informações extremamente útil é aquele obtido através dos cálculos de correlações. Segundo Ferreira et al. (2003), a correlação fenotípica fornece uma estimativa da influência conjunta de causas genéticas e ambientais na expressão de uma dada característica. Por sua vez, os valores de correlação genotípica (que corresponde à porção genética da correlação fenotípica) têm sido empregados para orientar programas de melhoramento genético, uma vez que eles refletem a 
fração da expressão fenotípica que é de natureza herdável. Segundo Casali et al. (1984), a determinação de parâmetros genéticos-estatísticos de várias características de interesse da cenoura poderiam ter contribuído para avanços ainda maiores no melhoramento de populações desta hortaliça permitindo o aumento da eficiência do processo de seleção. No entanto, estas informações ainda estão em grande parte ausentes para populações provenientes de germoplasma de cenoura adaptado para cultivo em regiões tropicais e subtropicais (Vieira, 1988).

Freqüentemente os programas de melhoramento genético de cenoura necessitam avaliar características de planta tais como formato, tamanho, uniformidade e rugosidade da raiz, que são importantes no aspecto comercial da cultura. Os caracteres comprimento, diâmetro e peso de raiz, número de folhas, comprimento de folhas e diâmetro de xilema estão intimamente relacionados com a produtividade e qualidade das raízes. Todas essas características são de crucial importância no desenvolvimento de novas populações de cenoura com melhor aptidão para o processamento e maior aceitação no mercado in natura brasileiro (Vieira et al., 2001). Diversos estudos envolvendo diferentes populações de cenoura, avaliadas em diferentes condições edafoclimáticas, têm sido implementados visando estimar os valores de herdabilidade (Korla et al., 1980; Brar \& Sukhija, 1980; Timin, 1991; Nunes 1991; Vieira, 2001), bem como os valores das correlações genotípicas, fenotípicas e ambientais (McCollum, 1971; Bhagchandani \& Choudhury, 1980; Nunes, 1991; Vieira et al., 2001; Singh, 2002; Bhathia et al., 2002) para a maioria da características citadas anteriormente. Embora estes estudos tenham fornecido importantes informações, as estimativas de herdabilidade e correlação estão tipicamente associadas a uma população específica, sob condições ambientais especiais.

Desta forma, objetivo do presente trabalho foi estimar parâmetros genéticos relacionados a alguns dos principais caracteres de planta (parte aérea e raiz) relevantes para o melhoramento da ce- noura, utilizando-se progênies derivadas da cultivar Brasília, avaliadas durante o período de verão no Distrito Federal. Esta região pode ser considerada como representativa das condições edafoclimáticas predominantes no Brasil Central.

\section{MATERIAL E MÉTODOS}

O experimento foi implementado no Centro Nacional de Pesquisas de Hortaliças, em terreno tipo Latossolo vermelho amarelo (LVA) distrófico (Embrapa, 1999). Foram utilizadas 69 progênies de meios-irmãos obtidas de uma população derivada da cultivar Brasília, que foram cultivadas em campo durante o verão de 1999/2000. Estas progênies foram dispostas no delineamento de blocos casualizados com duas repetições e parcelas de $2 \mathrm{~m}^{2}$, compostas por quatro linhas de 20 plantas cada. O sistema de cultivo utilizado foi semeadura nos canteiros com posterior desbaste, 30 dias após o semeio. Os canteiros foram estabelecidos (preparo de solo, correção de acidez de solo e adubação) de acordo com as recomendações técnicas da cultura. Empregou-se como adubação química, $200 \mathrm{~g}$ da fórmula 414-8 por $\mathrm{m}^{2}$ na ocasião do plantio. Em cobertura, utilizaram-se $40 \mathrm{~g}$ de sulfato de amônio por $\mathrm{m}^{2}$, logo após o desbaste. Os tratos culturais foram os comumente recomendados para esta hortaliça, ressaltando-se que nenhuma pulverização para controle de pragas ou doenças foi executada durante o ciclo de cultivo.

Na colheita (95 dias após o semeio), retirou-se uma amostra de 13 plantas competitivas de cada parcela, sendo que cada uma dessas plantas foi avaliada para os seguintes caracteres: (1) número de folhas por planta, correspondente ao número de folhas com no mínimo 5 cm de comprimento em cada planta; (2) comprimento da maior folha da planta, tomado do ponto de inserção na raiz até a extremidade do último folíolo; (3) comprimento da raiz, medido desde a inserção das folhas até a ponta da raiz; (4) peso da raiz, quantificado em balança de precisão de $1 \mathrm{~g}$; (5) diâmetro da raiz, medido com paquímetro na metade do comprimento da raiz e; (6) diâ- metro do xilema, medido com paquímetro na metade do comprimento da raiz. Em seguida, os dados individuais obtidos foram transformados para média da parcela e submetidos à análise de variância e covariância, utilizando-se o aplicativo computacional Genes-UFV (Cruz, 1997). Foram estimados os seguintes parâmetros:

Variância fenotípica entre médias de progênies: $\quad \hat{\sigma}_{f}^{2}=(Q M P / r)$, onde: $\hat{\sigma}_{f}^{2}=$ variância fenotípica, $r=$ repetições e $Q M P=$ quadrado médio das progênies;

Variância genotípica entre médias de progênies:

$$
\hat{\sigma}_{g}^{2}=(Q M P / Q M R) / r,
$$

onde: $\hat{\sigma}_{g}^{2}=$ variância genotípica, $Q M P=$ quadrado médio das progênies, $Q M R=$ quadrado médio do resíduo e $r=$ repetições;

Variância genética aditiva:

$$
\hat{\sigma}_{g}^{2}=1 / 4 . \hat{\sigma}_{a}^{2},
$$

onde $\hat{\sigma}_{a}^{2}=$ variância genética aditiva e $\hat{\sigma}_{g}^{2}=$ variância genotípica;

Variância ambiental média:

$$
\hat{\sigma}_{e}^{2}=(Q M R / r) \text {, }
$$

onde: $\hat{\sigma}_{e}^{2}=$ variância ambiental, $Q M R=$ quadrado médio do resíduo e $r=$ repetições;

Herdabilidade no sentido amplo (baseada na média das parcelas)

$$
\Rightarrow \hat{h}^{2}=\hat{\sigma}_{g}^{2} / \hat{\sigma}_{f}^{2},
$$

onde: $\hat{h}_{a}{ }^{2}=$ herdabilidade no sentido amplo, $\hat{\sigma}_{g}^{2}=$ variância genotípica e $\hat{\sigma}_{f}^{2}=$ variância fenotípica;

Coeficiente de variação genética:

$$
C \hat{V}_{g}=\left(\sqrt{\hat{\sigma}_{g}^{2}} / \hat{m}\right) \times 100,
$$

onde: $C \hat{V}_{g}=$ coeficiente de variação genética, = variância genotípica e corresponde a média geral do caráter. 
Tabela 1. Estimativas das variâncias fenotípicas $\left(\mathrm{V}_{\mathrm{f}}\right)$, genotípicas $\left(\mathrm{V}_{\mathrm{g}}\right)$, genética aditiva $\left(\mathrm{V}_{\mathrm{a}}\right)$, ambientais $\left(\mathrm{V}_{\mathrm{e}}\right)$, herdabilidade no sentido amplo $\left(\mathrm{h}_{\mathrm{a}}^{2}\right)$, coeficiente de variação genético $\left(\mathrm{CV}_{\mathrm{g}}\right)$ e razão entre coeficiente de variação genético e ambiental $\left(\mathrm{CV}_{\mathrm{g}} / \mathrm{CV}_{\mathrm{e}}\right)$, para os caracteres de planta. Brasília, Embrapa Hortaliças, 2000.

\begin{tabular}{lcccccc}
\hline Parâmetros & NFP & $\mathbf{C F}^{1}$ & $\mathbf{P R}^{1}$ & $\mathbf{C R}^{1}$ & $\mathbf{D R}^{1}$ & $\mathbf{D X}^{1}$ \\
\hline $\mathrm{V}_{\mathrm{f}}$ & 0,776878 & 10,364133 & 98,371904 & 1,166282 & 0,056855 & 0,014298 \\
$\mathrm{~V}_{\mathrm{g}}$ & 0,453067 & 8,040238 & 56,341657 & 0,497266 & 0,016985 & 0,008283 \\
$\mathrm{~V}_{\mathrm{a}}$ & 1,812268 & 32,160952 & 225,366628 & 1,989064 & 0,067940 & 0,033132 \\
$\mathrm{~V}_{\mathrm{e}}$ & 0,323811 & 2,323895 & 42,030247 & 0,669016 & 0,03987 & 0,006015 \\
$\mathrm{~h}_{\mathrm{a}}^{2} \%$ & 58,31 & 77,57 & 57,27 & 42,63 & 29,87 & 57,93 \\
$\mathrm{CV}$ & 9,79 & 6,96 & 10,96 & 5,22 & 4,87 & 7,71 \\
$\mathrm{CV}_{\mathrm{e}}$ & 11,79 & 5,31 & 13,53 & 8,7 & 10,58 & 9,40 \\
$\mathrm{CV}_{\mathrm{g}} / \mathrm{CV}$ & 0,83 & 1,31 & 0,81 & 0,60 & 0,46 & 0,82 \\
\hline
\end{tabular}

${ }^{1} \mathrm{NFP}=$ número de folhas médio por planta em cada parcela; $\mathrm{CF}=$ comprimento médio da maior folha; $\mathrm{PR}=$ peso médio da raiz por parcela; $\mathrm{CR}=$ comprimento médio da raiz por parcela; $\mathrm{DR}=$ diâmetro médio de raiz por parcela; $\mathrm{DX}=$ diâmetro médio do xilema da raiz por parcela.

Correlações fenotípicas:, onde: correlação fenotípica, $\mathrm{PMP}_{\mathrm{xy}}=$ produto médio das progênies/tratamentos, $\mathrm{QMP}_{\mathrm{x}}=$ quadrado médio do caráter $\mathrm{x}$ das progênies/tratamentos e $\mathrm{QMPT}_{\mathrm{y}}=$ = quadrado médio progênies/tratamentos total;

Correlações ambientais: , onde: correlação ambiental, $\mathrm{PMP}_{\mathrm{xy}}=$ produto médio do resíduo, $\mathrm{QMR}_{\mathrm{x}}=$ quadrado médio do resíduo do caráter $\mathrm{X}$ e $\mathrm{QMR}=$ = quadrado médio do resíduo do caráter Y;

Correlações genotípicas: , onde: correlação genotípica, estimador da covariância genética dos caracteres $\mathrm{X}$ e Y e e = estimadores da variância genéticas dos caracteres $\mathrm{X}$ e $\mathrm{Y}$, respectivamente.

\section{RESULTADOS E DISCUSSÃO}

Houve diferenças significativas entre as 69 famílias de meios-irmãos avaliadas, a $1 \%$ de probabilidade, pelo teste $\mathrm{F}$, para os caracteres número médio de folhas por planta (NFP), comprimento médio da maior folha $(\mathrm{CF})$, peso médio da raiz (PR), comprimento médio da raiz (CR), diâmetro médio de raiz (DR) e diâmetro médio do xilema da raiz (DX), evidenciando que existe variabilidade entre as famílias.

Os valores de herdabilidade no sentido amplo $\left(\mathrm{h}_{\mathrm{a}}^{2}\right)$ foram medianamente altos para todos os caracteres, exceto para DR, que foi de 29,9\% (Tabela 1). Valores de herdabilidade relativamente elevados para essas características em cenoura já haviam sido reportados anteriormente. Korla et al. (1980) relataram valores de herdabilidade da ordem de $64,3 \%$ e $90 \%$, respectivamente para PR e CF, similares aos verificados no presente estudo. Por sua vez, Brar \& Sukhija (1980) detectaram valores altos de herdabilidade no sentido amplo para CF (98\%), PR (85\%), DR (76\%) e CR (76\%). Em estudos conduzidos no Brasil, Vieira et al. (2001) constataram valores de herdabilidade para $\mathrm{CR}$ que variaram de $12 \%$ a $44 \%$ em progênies derivadas da cultivar Alvorada. As diferenças de valores de herdabilidade encontrados no presente estudo com aqueles reportados por Vieira et al. (2001), Brar \& Sukhija (1980) e Timin (1991) podem ser atribuídas à utilização de populações com diferentes bases genéticas.

Quanto à relação $\mathrm{CV}_{\mathrm{g}} / \mathrm{Cv}_{\mathrm{e}}$ (Tabela 1$)$ foram encontrados valores menores que a unidade para quase todos os caracteres estudados, evidenciando que a adoção exclusiva de métodos simples de melhoramento, como seleção massal, não reverterá em ganhos consideráveis, sendo recomendável o emprego de unidades de seleção estruturadas como, por exemplo, famílias. Segundo Vencovsky (1987), existe uma situação muito favorável para a obtenção de ganhos na seleção quando a relação $\mathrm{CV}_{\mathrm{g}} / \mathrm{CV}_{\mathrm{e}}$ tende a um $(1,0)$ ou maior que 1,0 , na medida em que, nesses casos, a variação genética supera a variação ambiental. Por este princípio, a seleção quando é praticada no primeiro ano maximiza o ganho genético, que vai paulatinamente diminuindo a partir dos anos subseqüentes. $\mathrm{O}$ maior valor da relação $\mathrm{CV}_{\mathrm{g}}$ $\mathrm{CV}_{\mathrm{e}}(1,31)$ foi observado para $\mathrm{CF}$ indi- cando que seleção para este caráter apresenta as condições mais favoráveis em termos de ganhos genéticos imediatos.

Os valores dos coeficientes de variação genética $\left(\mathrm{CV}_{\mathrm{g}}\right)$ para os caracteres estudados variaram de 4,87 a 10,96; enquanto os valores dos coeficientes de variação ambiental $\left(\mathrm{CV}_{\mathrm{e}}\right)$ variaram de 5,31 a 13,53. Entretanto, os valores dos coeficientes de variação ambiental foram baixos para maioria dos caracteres (Tabela 1), o que denota um bom controle ambiental, eficiência no desenho experimental e baixa variabilidade genética na população em estudo, exceto para a característica $\mathrm{CF}$.

O baixo valor de herdabilidade $\left(\mathrm{h}_{\mathrm{a}}^{2}\right)$ observado para o caráter DR (Tabela 1) pode ser decorrente do estreitamento genético após sucessivos ciclos de seleção visando o aumento de produtividade (peso de raiz= PR). O caráter DR apresenta alto valor de correlação genotípica com PR $(r=0,84)$, o que pode ter conduzido a redução considerável da variabilidade genética para estes atributos. É interessante comparar os resultados obtidos neste trabalho com os resultados obtidos com progênies da cultivar Brasília, avaliadas em Minas Gerais. No estudo realizado em Minas Gerais, as estimativas de herdabilidade para DR, CR e DX foram da ordem de $53,7 \%, 61,6 \%$ e $31,8 \%$, respectivamente (Nunes, 1991). Vale ressaltar que o valor mais elevado de herdabilidade $(\mathrm{CF}=77,6 \%)$ pode ser explicado, em parte, pela ausência de inclusão deste atributo como um dos objetivos básicos nos programas de melhoramento genético de cenoura conduzidos no Brasil. 
Tabela 2. Matriz de correlações fenotípicas $\left(r_{F}\right)$, genotípicas $\left(r_{G}\right)$ e ambientais $\left(r_{A}\right)$ entre caracteres de planta de cenoura de 69 famílias de meios-irmãos provenientes da cultivar Brasília. Brasília, Embrapa Hortaliças, 2000.

\begin{tabular}{|c|c|c|c|c|c|c|}
\hline Caracteres & $\mathbf{r}$ & $\mathrm{CF}^{1}$ & $\mathbf{P R}^{1}$ & $\mathrm{CF}^{1}$ & $\mathrm{DR}^{1}$ & $\mathbf{D X}^{1}$ \\
\hline & $\mathrm{F}$ & 0,27 & $0,30^{*}$ & 0,26 & 0,25 & $-0,07$ \\
\hline \multirow[t]{3}{*}{$\mathrm{NFP}^{1}$} & G & $0,34^{*}$ & $0,49^{*}$ & $0,45^{*}$ & 0,28 & $-0,25$ \\
\hline & A & 0,12 & 0,03 & 0,07 & 0,25 & 0,16 \\
\hline & $\mathrm{F}$ & & 0,18 & 0,02 & 0,03 & 0,07 \\
\hline \multirow[t]{3}{*}{$\mathrm{CF}^{1}$} & G & & 0,28 & 0,05 & 0,07 & 0,11 \\
\hline & A & & $-0,01$ & $-0,02$ & 0,00 & 0,00 \\
\hline & $\mathrm{F}$ & & & $0,54^{*}$ & $0,53^{*}$ & $0,50^{*}$ \\
\hline \multirow[t]{3}{*}{$\mathrm{PR}^{1}$} & G & & & $0,50^{*}$ & $0,84^{*}$ & $0,36^{*}$ \\
\hline & A & & & $-0,59^{*}$ & $-0,33^{*}$ & $-0,708$ \\
\hline & $\mathrm{F}$ & & & & 0,12 & 0,12 \\
\hline \multirow[t]{3}{*}{$\mathrm{CR}^{1}$} & G & & & & 0,18 & $-0,13$ \\
\hline & A & & & & $-0,09$ & $-0,39^{*}$ \\
\hline & $\mathrm{F}$ & & & & & $0,34^{*}$ \\
\hline \multirow[t]{2}{*}{$\mathrm{DR}^{1}$} & G & & & & & $0,30^{*}$ \\
\hline & A & & & & & $-0,40^{*}$ \\
\hline
\end{tabular}

*Significativo ao nível de $1 \%$ de probabilidade pelo teste $\mathrm{t} ;{ }^{1} \mathrm{NFP}=$ número de folhas médio por planta em cada parcela; $\mathrm{CF}=$ comprimento médio da maior folha; $\mathrm{PR}=$ peso médio da raiz por parcela; $\mathrm{CR}=$ comprimento médio da raiz por parcela; $\mathrm{DR}=$ diâmetro médio de raiz por parcela; $\mathrm{DX}=$ diâmetro médio do xilema da raiz por parcela.

Foram calculadas ainda as correlações fenotípica, genotípica e ambiental para os seis caracteres avaliados (Tabela 2). A mencionada magnitude do valor de correlação genotípica positiva entre PR e DR $(0,84)$ foi muito próxima ao valor encontrado por McCollum (1971) para este par de caracteres $(\mathrm{r}=$ 0,87). Vieira et al. (2001) também encontraram valor similar $(\mathrm{r}=0,90)$ em progênies da cultivar Alvorada. Singh (2002), avaliando valores de correlação em caracteres de raiz de cenoura, verificou a ocorrência de coeficientes de correlação genotípica alta e positiva entre comprimento e peso de raiz. Estes resultados são de certa maneira esperados uma vez que peso de raiz é uma característica dependente, dentre outros atributos, da largura e comprimento da raiz.

A estimativa do valor de correlação genotípica negativa observado entre NFP e DX $(r=-0,25)$, poderia ser decorrente da existência de pleiotropia ou de alelos ligados em fase de repulsão. É interessante também notar que a magnitude no valor da correlação genotípica para NFP e DX parece ser uma característica peculiar do germoplasma tropical brasileiro de cenoura uma vez que valores relativamente próximos $(\mathrm{r}=$ $0,15$ e $r=-0,28)$ foram também encon- trados em trabalhos independentes conduzidos por Nunes (1991) e por Vieira et al. (2001), respectivamente. Vale ressaltar que a existência de correlação genotípica negativa entre os pares de caracteres (CR $x$ DX) indicam a possibilidade de desenvolvimento de populações com qualidade de raiz adequada para processamento mínimo visando a produção das chamadas mini-cenouras ("baby-carrots") a partir de populações derivadas da cultivar Brasília. Para alcançar boa qualidade e rendimento na produção de mini-cenouras, busca-se combinar menor diâmetro de xilema e maior comprimento de raiz.

Os valores de correlação genotípicas (Tabela 2) envolvendo (NFP $x \mathrm{CF}$ ), (NFP $x$ PR), (NFP $x$ CR) e (NFP $x$ DR) são similares àqueles encontrados por Vieira et al. (2001) para esses mesmos pares de caracteres na cultivar Alvorada. Adicionalmente, os valores relativos a correlação genotípica entre NFP e PR (Tabela 2) são similares aos encontrados por Bhathia et al. (2002). Já a estimativa de correlação genotípica entre DR e PR (Tabela 2) foi similar ao resultado encontrado por McCollum (1971), da ordem de 0,87 .

Bhagchandani \& Choudhury (1980) constataram alta correlação genotípica positiva entre DR e DX ( $r=0,97)$, diferentemente do resultado obtido neste trabalho, $r=0,30$ (Tabela 2). Esse fato pode ser decorrente do diferente grau de melhoramento das populações utilizadas em cada tipo de estudo. Adicionalmente, vale destacar os sinais negativos de algumas correlações ambientais entre pares de caracteres indicam que o ambiente afeta um caráter em detrimento do outro (Tabela 2). Além disso, verificam-se combinações de pares de caracteres em que os sinais das correlações genotípicas e ambientais são diferentes, indicando que as causas de variação genética e ambiental presumivelmente influenciaram os caracteres por meio de diferentes mecanismos fisiológicos (Falconer, 1981).

Os valores de herdabilidade e correlações estabelecidos em outras populações, sob outras circunstâncias de cultivo, podem ser semelhantes se a estrutura genética da população e/ou as condições de ambiente não apresentarem grandes variações (Vieira, 1988). Desta forma, o acúmulo de conhecimento sobre os parâmetros genéticos associados a estas características em populações da cultivar Brasília pode permitir o estabelecimento estratégias de melhoramento mais efetivas visando desenvolver cultivares superiores para macro-regiões cujas condições ambientais sejam relativamente uniformes, tais como o Planalto Central do Brasil.

\section{REFERÊNCIAS}

BHAGCHANDANI PM; CHOUDHURY B. 1980. Correlations and path coefficient studies in carrot. Indian Journal of Agricultural Science 50: 663-666.

BHATHIA MK; BASWANA KS; DHARAMVEER DUHAN; DUHAN D. 2002. Correlation and path coefficient analysis in carrot (Daucus carota L.). Haryana Journal of Horticultural Sciences 31: 227-229.

BRAR JS; SUKHIJA BS. 1980. Variability, heritability and genetic advance in carrot (Daucus carota L.). Journal of Research Punjab Agriculture University 17: 442-443.

BUSO JA. 1978. Estimativas de Parâmetros Genéticos de Caracteres de Planta e Bulbo de Cebola (Allium cepa L.). Piracicaba: ESALQ. 132p. (Tese mestrado).

CASALI VWD; PINTO CMF; PADUA JG. 1984 Origem e botânica da cenoura. Informe Agropecuário 10: 8-9.

CRUZ CD. 1997. Programa Genes: Aplicativo Computacional em Genética e Estatística. Viçosa: UFV. 442p. 
EMBRAPA. Centro Nacional e Pesquisa de Solos. 1999. Sistema Brasileiro de Classificação de Solos. Brasília: Embrapa-SID. 412p.

FALCONER DS. 1981. Introdução à Genética Quantitativa. Viçosa: UFV. 279p.

FERREIRA MAJF; QUEIROZ MA; BRAZ LT; VENCOVSKY R. 2003. Correlações genotípicas, fenotípicas e de ambiente entre dez caracteres de melancia e suas implicações para o melhoramento genético. Horticultura Brasileira 21: 438-441.

HALLAUER AR, MIRANDA FILHO JB. 1981. Quantitative Genetics in Maize Breeding. Ames, Iowa State University Press. 468p.

KORLA BN; SINGH AK; PATTAN RS. 1980. A research note on variability studies in carrot. Punjab Horticulture Journal 20: 215-217.

McCOLLUM GO. 1971. Greening of carrot roots (Daucus carota L.): Estimates of heritability and correlation. Euphytica 20: 549-560.
NUNES MUC. 1991. Estimativas de Parâmetros Genéticos e de Correlações de Caracteres da Raiz, Parte Aérea e Sementes de Progênies de Meio-irmãos da Cenoura (Daucus carota L.) cv. Brasília. Viçosa: UFV. 133p. (Tese doutorado).

RUBATZKY VE; QUIROS CF; SIMON PW 1999. Carrots and Related Vegetable Umbelliferae. Crop Production Science in Horticulture. CABI Publishing, CAB International, Wallingford, United Kingdom 10: 294.

SINGH B; KUMAR D; KUMAR A; GEETA SINGH G; SINGH G. 2002. Correlation studies in carrot (Daucus carota L.). Progressive Agriculture 2: 84-85.

TIMIN NI. 1991. Genotypic variation and the heritance of characters in carrot. Plant Breeding Abstracts 61: 482 (Resumo de trabalho original em russo).
VENCOVSKY R. 1987. Herança Quantitativa. In: PATERNIANI E; VIEGAS GP. Melhoramento e Produção de Milho no Brasil. 2 ed. Campinas: Fundação Cargill. p. 137-214.

VIEIRA JV. 1988. Herdabilidades, Correlações e Índice de Seleção em População de Cenoura (Daucus carota L. ). Viçosa: UFV. 86p. (Tese doutorado).

VIEIRA JV, DELLA-VECCHIA PT; IKUTA H. 1983. Cenoura 'Brasilia'. Horticultura Brasileira 1: 42.

VIEIRA JV; BOITEUX LS; ARAGÃO FAS. 2001. Estimativas de parâmetros genéticos relativos ao comprimento de raízes de cenoura em populações derivadas da cultivar Alvorada. In: CONGRESSO BRASILEIRO DE OLERICULTURA, 41. Resumos... Brasília: SOB (CD-ROM). 